\title{
Presentación del monográfico
}

\author{
Raquel HERNÁNDEZ-GARCÍA* \\ Editora invitada del número especial \\ Directora I Congreso Nacional de Entrenadores de Judo \\ Facultad Ciencias del Deporte. Universidad de Murcia (España)
}

El judo es uno de los deportes más practicados en España, con más de 100 mil licencias, detrás de fútbol, baloncesto, caza, golf y montaña, y más de 1.000 clubes federados distribuidos por todo el territorio del estado (CSD, 2017). El hecho de que exista tanta práctica en nuestro país lleva aparejada la existencia de un gran volumen de profesionales del judo (entrenadores, profesores, monitores) que hacen posible que este deporte ocupe un lugar tan destacado en el ranking de deportes con mayor número de licencias.

Sin embargo, a pesar de disfrutar de estas cifras, son escasas las actuaciones formativas para el colectivo de los profesionales del judo, las cuales, salvo excepciones (véanse por ejemplo Mateo-Cubo \& Montero-Carretero, 2017; PulidoGonzález, 2013) se limitan a eventos autonómicos puntuales de actualización en temáticas muy específicas. Por ello, la mayoría de los entrenadores de judo desarrollan su trabajo tras un curso de formación para obtenerla titulación y el resto de formaciones que realizan son llevada a cabo desde una perspectiva eminentemente autodidacta.

Principalmente por este motivo, hace apenas un año se desarrolló el I Encuentro Nacional de Entrenadores de Judo, a través del Judo Club Ciudad de Murcia, con el principal objetivo de de generar un punto de encuentro entre profesionales y poder compartir conocimiento, experiencias y reflexiones. Tras el éxito de participación y la recogida de las valoraciones realizadas por los participantes, el equipo organizativo se decidió a dar un paso más y convertir la segunda edición en el I Congreso Nacional de Entrenadores de Judo, esta vez vehiculado a través de la Facultad de Ciencias del Deporte de la Universidad de Murcia. En esta ocasión el eje y objetivo principal del evento será fomentar la transferencia de conocimiento desde la ciencia hacía la práctica y viceversa.

Para ello, se desarrollara un congreso de 13 horas donde se contará con cinco ponentes nacionales de elevado nivel: Sergio Domenech, Marcial Romero, Edu Carvalleira, Yolanda Soler y Carlos Fernandez; una mesa redonda sobre el "Papel del entrenador en España", un taller de "Primero auxilios en el tatami" y un espacio abierto para envió de

\section{Comité Organizador}

Facultad Ciencias del Deporte de la Universidad de Murcia (San Javier)

- Raquel Hernández García

- Arturo Díaz Suarez

- Jose María López Gullón

- Enrique Ortega Toro

- Marcelino García Mercader

\section{Comité Científico}

- Vicente Carratalá Deval (Presidente) -Universidad de Valencia

- Raquel Escobar Molina Universidad de Granada

- Carlos Montero Carretero Universidad Miguel Hernández del Elche

- Gema Torres Luque Universidad de Jaén

- Carlos Gutierrez GarcíaUniversidad de León

- Edu Carballeira Fernández Grupo Investigación Performance \& Health (UDC)

- Antonio Garcíade Alcaraz Serrano- Universidad Isabel I

- Roberto Ruiz BarquínUniversidad Autónoma de Madrid

- Juan Bonitch GóngoraUniversidad de Granada

- Andrés Robles Rodriguez Centro Universitario San Isidro (Universidad Pablo Olavide)

\section{Patronazgo}

- Universidad de Murcia

- Facultad Ciencias del Deporte San Javier (Murcia)

- Federación de la Región de Murcia de Judo y Deportes Asociados.

\section{*Email: rhernandezgarcia@um.es}


trabajos científicos y experiencias prácticas.

La edición especial que ustedes ya pueden consultar está compuesta por los nueve resúmenes aceptados para exposición de trabajos científicos y prácticos, presentados por un total de 23 profesores e investigadores de judo del ámbito nacional. Esperamos que este tipo de iniciativas tengan una buena acogida y que puedan contribuir positivamente al desarrollo de nuestro querido deporte.
- Tándem - Técnicos Deportivos S.L

- Revista de Artes Marciales Asiaticas

\section{Referencias}

Consejo Superior de Deportes. (2017). Memoria 2016/ Licencias y Clubes federados. Recuperado el 31/05/2018, de http://www.csd.gob.es/csd/estaticos/asoc-fed/LicenciasyClubes-2016.pdf

Mateo-Cubo, F., \& Montero-Carretero, C. (2017). Informe sobre el I Seminario Internacional Ciencia en Judo (SICJ). Revista de Artes Marciales Asiáticas, 12(2), 106-111. doi: $\underline{10.18002 / \text { rama.v12i2.5193 }}$

Pulido-González, J. (2013). Informe sobre el I Congreso Científico de Judo Escuela De Frutos Universidad Miguel Hernández con Campo de Entrenamiento. Revista de Artes Marciales Asiáticas, 8(2), 466-470. doi: 10.18002/rama.v8i2.949 